\title{
CRISPR technology is revolutionizing the improvement of tomato and other fruit crops
}

\author{
Tian Wang ${ }^{1}$, Hongyan Zhang $^{2}$ and Hongliang Zhu (1) $^{3}$
}

\begin{abstract}
Fruits are major sources of essential nutrients and serve as staple foods in some areas of the world. The increasing human population and changes in climate experienced worldwide make it urgent to the production of fruit crops with high yield and enhanced adaptation to the environment, for which conventional breeding is unlikely to meet the demand. Fortunately, clustered regularly interspaced short palindromic repeat (CRISPR) technology paves the way toward a new horizon for fruit crop improvement and consequently revolutionizes plant breeding. In this review, the mechanism and optimization of the CRISPR system and its application to fruit crops, including resistance to biotic and abiotic stresses, fruit quality improvement, and domestication are highlighted. Controversies and future perspectives are discussed as well.
\end{abstract}

\section{Introduction}

Fruits are major sources of fibers, vitamins, and minerals worldwide ${ }^{1}$. In some parts of Asia, Africa, and South America, banana, breadfruit, and date fruit also serve as staple foods ${ }^{2-4}$. Fruit crops are at high risk under climate change ${ }^{5}$. To increase the chances of a steady fruit supply, our ancestors domesticated wild plant species into cultivated crops. Following the "rediscovery" of Mendel's laws in 1900, breeders started selecting and crossing superior plants ${ }^{6}$. However, conventional breeding has major shortcomings. First, it largely depends on existing natural allelic variations and is thus inefficient for obtaining the desired characteristics by random mixing of tens of thousands of genes ${ }^{5}$. Although conventional breeding has increased crop productivity, it is often accompanied by loss of fitness and genetic diversity ${ }^{7}$, and it is a rather time-consuming practice that could hardly

\footnotetext{
Correspondence: Hongliang Zhu (hlzhu@cau.edu.cn)

${ }^{1}$ College of Food Science and Nutritional Engineering, China Agricultural University, 100083 Beijing, China

${ }^{2}$ Key Laboratory of Food Nutrition and Safety of Shandong Normal University, College of Life Science, Shandong Normal University, 250014 Jinan, China Full list of author information is available at the end of the article.
}

ensure a sufficient food supply for the rapidly growing human population around the world ${ }^{8}$. Therefore, continuous technological innovation is required to meet the increasing demands of consumers?

Genetic engineering techniques have numerous applications in fruit crops, as they allow improvement of important agronomic traits such as biotic and abiotic stress tolerance and fruit quality. During the past two decades, several fruit crops have been modified using these techniques. In contrast to conventional breeding, recombinant DNA technology allows transfer of the desired genes from any organism, plant or microorganism into fruit crops, extending the opportunities for fruit yield enhancement by offering new genotypes and phenotypes for breeding purposes, and ultimately improving fruit quality as well as enhancing shelf life. Thus, genetic engineering has been ranked as the fastest developing technology in agriculture ${ }^{10}$. The organisms obtained by recombinant DNA technology are termed "genetically modified" (GM). In 1994, the transgenic "Flavr Savr tomato" was approved for commercial growth in the United States (US) by the Food and Drug Administration (FDA). The modification it contained allowed a slowing of

\section{(c) The Author(s) 2019}

(c) (i) Open Access This article is licensed under a Creative Commons Attribution 4.0 International License, which permits use, sharing, adaptation, distribution and reproduction cc) in any medium or format, as long as you give appropriate credit to the original author(s) and the source, provide a link to the Creative Commons license, and indicate if changes were made. The images or other third party material in this article are included in the article's Creative Commons license, unless indicated otherwise in a credit line to the material. If material is not included in the article's Creative Commons license and your intended use is not permitted by statutory regulation or exceeds the permitted use, you will need to obtain permission directly from the copyright holder. To view a copy of this license, visit http://creativecommons.org/licenses/by/4.0/. 
its ripening process and prevented it from softening after picking. The GM papaya authorized for marketing can resist ring spot virus attacks and show enhanced productivity. Eighty percent of Hawaiian papaya produced today is genetically engineered, and no alternative method is available ${ }^{11}$.

However, the development of new GM crops is largely affected by regulatory-approval processes because the purpose of the approval system is preventing harm to human health and the environment, as well as avoiding economic losses ${ }^{12}$. These regulations also help ensure consumer confidence in GM crop biosafety ${ }^{13}$. As a result, the costs of obtaining approval for new GM crops can be very high, and the regulatory requirements may also delay product marketing ${ }^{14}$. Jefferson et al. ${ }^{15}$ have argued that these stringent regulations can result in unnecessary barriers to the introduction of new GM crops. Thus, clustered regularly interspaced short palindromic repeat (CRISPR) technology may be a better choice: in 2016, a CRISPR-edited mushroom escaped US regulation as it fell outside the GM organisms legislation by not containing foreign $\mathrm{DNA}^{16}$. In 2017, the FDA approved the marketing of a false flax with increased oil content and a droughttolerant soybean ${ }^{17}$, indicating that the CRISPR-edited crops were not under the same stringent regulations as traditional GM crops and that the CRISPR technology would definitely revolutionize the pace of crop breeding ${ }^{18}$.

\section{Genome editing has been revolutionized by the development of CRISPR technology}

The discovery of CRISPR in the prokaryote immune system

The CRISPR system is a sophisticated adaptive immune mechanism present in bacteria and Archaea for defense against invading bacteriophages and exogenous plasmids ${ }^{19}$. It was first discovered in the genome of Escherichia coli in $1987^{20}$ and officially named by the Dutch scientist who identified CRISPR-associated (Cas) genes ${ }^{21}$. In 2005, three different research groups simultaneously found that the short sequences of many CRISPR spacers were highly homologous with sequences originating from extra chromosomal $\mathrm{DNA}^{22-24}$, indicating a relationship between CRISPR and specific immunity. Nearly a decade later, CRISPR-Cas was successfully engineered into an efficient tool to edit human, animal, and plant genomes $^{25,26}$, extensively boosting its application in fields as diverse as pharmacology, animal domestication, and food science $^{27}$.

A complete CRISPR-Cas locus comprises a CRISPR array that harbors short repetitive elements intercalated with invader DNA-targeting spacers, an AT-rich leader sequence, and an operon of Cas genes encoding the Cas proteins ${ }^{28}$. Based on the different participating Cas proteins, CRISPR-Cas systems can be categorized into three main types: type I and type III systems use a large multi-
Cas protein complex for binding and targeting ${ }^{29,30}$, while the type II system requires only a single protein, the CRISPR-associated protein 9 (Cas9), for RNA-guided double-stranded DNA recognition and cleavage using its two distinct domains, RuvC and $\mathrm{HNH}^{31}$. The simplicity of the type II CRISPR (i.e., of the CRISPR-Cas9 system) enabled remarkable progress in genome engineering ${ }^{32}$.

\section{The mechanism of CRISPR-Cas9}

In general, the action of the CRISPR-Cas9 system can be divided into three stages in response to invading foreign DNA $^{33,34}$ : (i) acquisition stage-the invading DNA is identified and a spacer sequence derived from the target DNA is inserted into the host CRISPR array to establish immunological memory; (ii) expression stage-the Cas9 protein is expressed, and the CRISPR array is transcribed into a precursor RNA transcript (pre-crRNA). A noncoding trans-activating CRISPR RNA (crRNA) then hybridizes to the pre-crRNA and Cas9 protein and processes them into mature RNA units known as crRNAs; and (iii) interference stage-the mature crRNA guides the Cas9 protein to recognize the appropriate DNA target, leading to the cleavage and degradation of the invading foreign DNA.

The Cas9 protein cuts the DNA to generate a doublestrand break (DSB), triggering cellular DNA repair mechanisms (Fig. 1). In the absence of a homologous repair template, the error-prone non-homologous endjoining (NHEJ) pathway is activated and introduces random insertions/deletions or even substitutions at the DSB site, generally resulting in the disruption of gene function. Alternatively, if donor DNA template homologous to the sequence surrounding the DSB site is available, the errorfree homology-directed repair (HDR) pathway is initiated, leading to mutations that perform precise gene modification, including gene knock-in, deletion, or mutation ${ }^{35}$. At present, the most commonly used Cas9 protein comes from Streptococcus pyogenes $(S p)^{36}$. To exploit this system for genome editing, synthetic single-guide RNAs (sgRNAs) are required to construct the CRISPR-Cas9 expression cassettes. The Cas9 protein is then guided to specific genomic sites by the sgRNAs that recognize the NGG-type protospacer adjacent motif and targets DNA sequences through Watson-Crick base pairing ${ }^{37}$ (Fig. 1).

\section{The optimization of the CRISPR-Cas system in plants}

Since the CRISPR-Cas system was successfully engineered to edit plant genomes in 2013, numerous efforts have been made to transform it into a more powerful tool. At present, CRISPR-Cas has multiplex editing capability, that is, it edits more than one gene at a time ${ }^{38}$. In addition, CRISPR-Cas can target not only the open reading frame $(\mathrm{ORF})^{39}$ and untranslated region ${ }^{40}$ of one coding gene but also non-coding RNAs (ncRNAs) including long ncRNA ${ }^{41}$ 


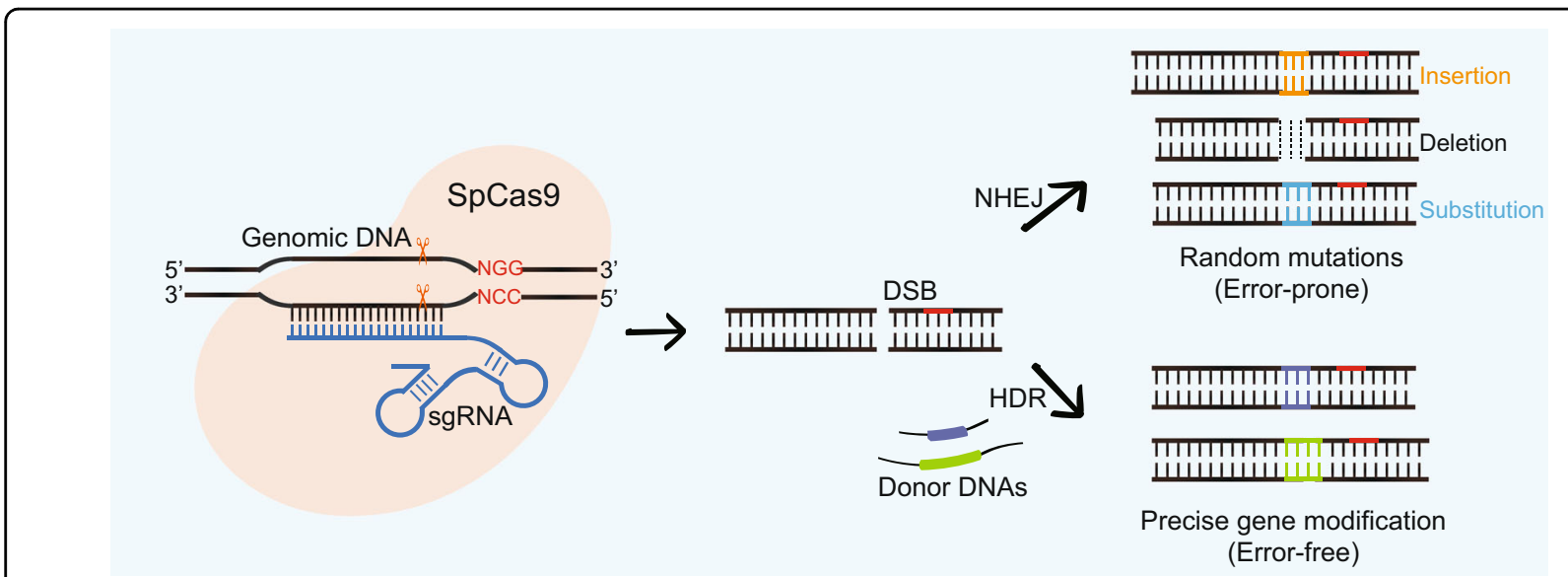

Fig. 1 The mechanism of CRISPR-Cas9-mediated genome engineering in plants. The sgRNA directs the SpCas9 protein to bind genomic DNA through a 20-nucleotide sequence and further guides it to introduce a DSB. This DSB causes random mutations when repaired by the error-prone NHEJ pathway or precise gene modification when repaired by the error-free HDR pathway. CRISPR, clustered regularly interspaced short palindromic repeat; Cas, CRISPR-associated; DSB, double-strand break; HDR, homology-directed repair; NHEJ, non-homologous end-joining; sgRNA, singleguide RNA

and microRNA ${ }^{42}$, as well as promoter regions ${ }^{43}$. Singlebase substitutions at genomic targets without requiring DSBs have also been achieved ${ }^{44}$. Here, we describe the optimization of the CRISPR-Cas system regarding the diversified development of Cas proteins, the optimization of Cas promoters, and the empowerment of sgRNAs with multiplexing capability (Table 1).

\section{Applications of CRISPR-Cas9 in fruit crops}

Duane Green has defined a fruit crop as a perennial, edible crop where the economic product is the true botanical fruit or derived from $\mathrm{it}^{81}$. Some plants, grown primarily as annuals, such as tomatoes, cucumbers, and melons, are also considered fruit $\mathrm{crops}^{82}$. Due to its easily achieved germplasm resources, simple diploid inheritance, efficient breeding, short growing period, ease of genetic transformation, and extensive research, tomato acts as a model for fruit biology ${ }^{1}$. Here, we summarize the applications of the CRISPR-Cas9 system in tomato and other fruit crops (Fig. 2 and Table 2).

\section{Current applications of CRISPR-Cas9 in tomato}

In 2014, the CRISPR-Cas9 system was first applied in tomato. Argonaute 7 was knocked out resulting in wiry phenotypes; the first leaves of mutants had leaflets without petioles and subsequently formed leaves lacking laminae ${ }^{127}$. Since then, numerous publications on CRISPR-Cas9 application in tomato have been published. We classified these publications into the following four groups: resistance to biotic stresses, resistance to abiotic stresses, improvement of tomato fruit quality, and domestication of tomato.

\section{Resistance to biotic stresses}

Biotic stresses include viruses, bacteria, fungi, and insects, all of which can attack plants and cause damage $^{128}$. CRISPR-Cas9 technology has been employed to obtain disease-resistant plants ${ }^{129}$ since its successful application for obtaining stable transgenic lines in 2013. Since then, CRISPR-Cas9 has been used against viral, fungal, and bacterial infection, which causes severe losses in tomato ${ }^{130,131}$.

For viruses, two strategies have been used. One consists of designing sgRNAs and targeting the virus genome directly through sequence complementation, and the other consists of modifying the tomato genes that confer antiviral characteristics. Tashkandi et al. ${ }^{83}$ used the CRISPR-Cas9 system to engineer tomato plants resistant to the tomato yellow leaf curl virus by targeting the coat protein and replicase loci of the genome. The transgenic tomato showed efficient viral interference and accumulated less viral genomic DNA than the wild-type (WT) plants. This kind of immunity remained active across multiple generations, indicating the utility of the CRISPRCas9 system for cultivating durable virus resistance plants. CRISPR-Cas9 technology has also been used to knock out crucial genes involved in resistance pathways, aiming to test whether these genes can confer immunity against viruses. Tomato Dicer-like 2 (DCL2) genes were targeted, and the $d c l 2$ mutants displayed viral symptoms when infected by potato virus $\mathrm{X}$, tobacco mosaic virus, and tomato mosaic virus, suggesting that $D C L 2$ is involved in the defense mechanism against RNA viruses ${ }^{84,85}$.

Fungi are accountable for multiple diseases, including mildew, smut, rust, and rot, which can cause dramatic 
Table 1 Optimization of the CRISPR-Cas system in plants

\begin{tabular}{|c|c|c|c|c|}
\hline Name & From & Function & Crop species & Refs. \\
\hline \multicolumn{5}{|l|}{ Cas proteins } \\
\hline St1Cas9 & Streptococcus thermophilus & $\begin{array}{l}\text { Size is smaller; recognizes longer PAMs ("NNAGAA" } \\
\text { or "NNGGAA") }\end{array}$ & Arabidopsis & 45 \\
\hline SaCas9 & Staphylococcus aureus & $\begin{array}{l}\text { Size is smaller; recognizes longer PAMs ("NNGGGT" } \\
\text { or "NNGAA") }\end{array}$ & Arabidopsis; tobacco & 45,46 \\
\hline SpCas9-VQR & Streptococcus pyogenes & Recognizes "NGA" PAM & Rice & 47 \\
\hline SpCas9- VRER & Streptococcus pyogenes & Recognizes "NGCG" PAM & Rice & 47 \\
\hline Cas12a (Cpf1) & $\begin{array}{l}\text { Acidaminococcus sp. BV3L6 (As); } \\
\text { Francisellanovicida }(F n) ; \\
\text { Lachnospiraceae bacterium } \\
\text { ND2006 }(L b)\end{array}$ & $\begin{array}{l}\text { Recognizes "TTTN" or "TTN" PAMs; targets DNA to } \\
\text { introduce a } 5^{\prime} \text { overhang; guided by a shorter } \\
\text { crRNA; exhibits little off-target activity }\end{array}$ & $\begin{array}{l}\text { Arabidopsis; maize; rice; } \\
\text { soybean; tobacco }\end{array}$ & $48-51$ \\
\hline Cas13a (C2c2) & Leptotrichiashahii & Targets single-stranded RNA with PFS of $A, U$, or $C$ & Rice; tobacco & 52,53 \\
\hline nCas9 & Streptococcus pyogenes & $\begin{array}{l}\text { Cas9 nickase contains a mutation in either of the two } \\
\text { nuclease domains of Cas9 protein. It induces SSBs }\end{array}$ & Arabidopsis; rice; tomato & $54-56$ \\
\hline dCas9 & Streptococcus pyogenes & $\begin{array}{l}\text { Deficient Cas9 contains mutations in both nuclease } \\
\text { domains of Cas9 protein. without cleavage activity. } \\
\text { The dCas9-based regulator can be developed when } \\
\text { fused with transcriptional activators or repressors }\end{array}$ & $\begin{array}{l}\text { Arabidopsis; maize; rice; } \\
\text { tobacco; wheat }\end{array}$ & $56-59$ \\
\hline Promoters & & Preferential expression & Crop species & Refs. \\
\hline \multicolumn{5}{|l|}{ Cas promoters } \\
\hline YAO & & $\begin{array}{l}\text { Tissues undergoing active cell division including the } \\
\text { shoot apical and root meristem, embryo sac, embryo, } \\
\text { endosperm, and pollen }\end{array}$ & Arabidopsis; citrus & 60,61 \\
\hline$S P L$ & & Sporogenous cells and microsporocytes & Arabidopsis & 62 \\
\hline EC1.1/EC1.2 & & Egg cells and one-cell stage embryos & Arabidopsis & 63,64 \\
\hline ICU2 & & Meristematic regions & Arabidopsis & 65 \\
\hline EFla, hisH4 & & Meristematic and reproductive tissues & Arabidopsis & 66 \\
\hline MGE & & Meiosis stage & Arabidopsis & 67 \\
\hline DMC1 & & Meiocytes & Arabidopsis; maize & 68,69 \\
\hline RPS5A & & At all developmental stages & Arabidopsis & 70 \\
\hline Strategy & & & Crop species & Refs. \\
\hline \multicolumn{5}{|l|}{ sgRNAs } \\
\hline \multicolumn{3}{|c|}{ Assemble multiple sgRNA expression cassettes into CRISPR-Cas vector } & $\begin{array}{l}\text { Arabidopsis; maize; Populus; } \\
\text { rice; tobacco; tomato }\end{array}$ & $71-75$ \\
\hline \multicolumn{3}{|c|}{ Produce numerous sgRNAs from a single polycistronic gene via the endogenous tRNA-processing system } & $\begin{array}{l}\text { Maize; potato; rice; } \\
\text { tomato; wheat }\end{array}$ & $76-80$ \\
\hline
\end{tabular}

PAM protospacer adjacent motif, sgRNA single-guide RNA, CRISPR-Cas clustered regularly interspaced short palindromic repeat-CRISPR-associated, tRNA transfer RNA, PFS protospacer flanking sequence, SSBs single-strand breaks, CrRNA CRISPR RNA

losses in crop yield and quality ${ }^{130}$. Downy and powdery mildews inflict severe economic losses in tomato. In Arabidopsis thaliana, downy mildew resistant 6 (DMR6), which belongs to the 2-oxoglutarate Fe(II)-dependent oxygenase superfamily, participates in salicylic acid homeostasis, and its overexpression results in enhanced susceptibility to downy mildew ${ }^{132}$. Researchers have used the CRISPR-Cas9 system to inactivate the DMR6 ortholog in tomato and found that $d m r 6$ mutants showed disease resistance against various pathogens, including Pseudomonas syringae, Phytophthora capsica, and Xanthomonas spp., without significant detrimental effects ${ }^{86}$. Mildew 


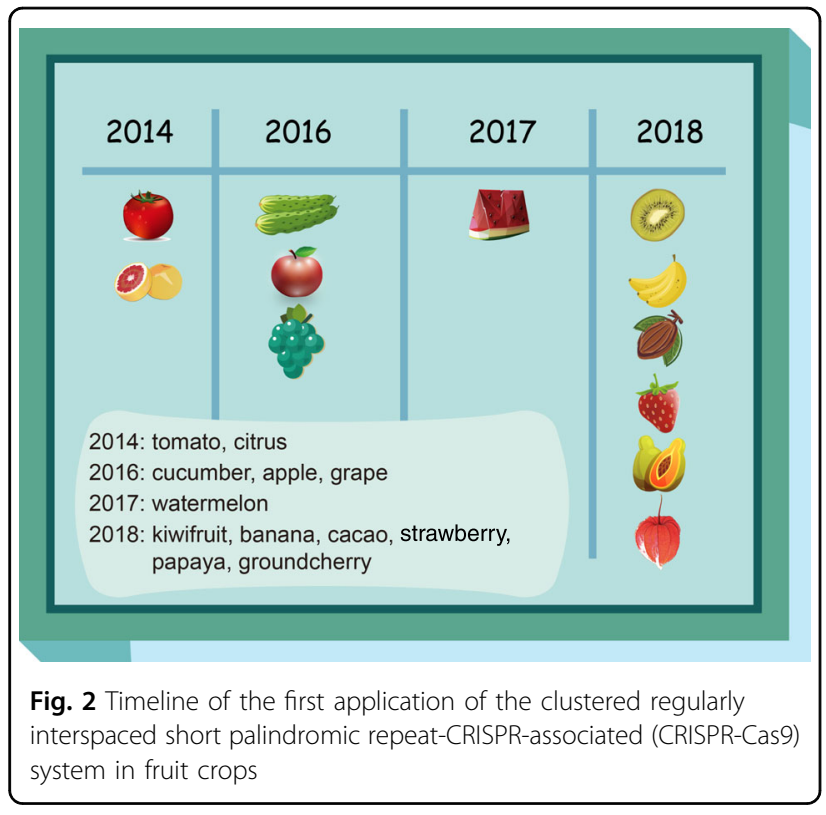

resistant locus $O 1$ (Mlo1), which encodes a membraneassociated protein, confers susceptibility to the fungi causing powdery mildew disease. Nekrasov et al. ${ }^{87}$ generated the tomato loss-of-function mlo1 mutant using CRISPR-Cas9 technology and found that the mutant was fully resistant to the powdery mildew fungus Oidium neolycopersici. Notably, the authors segregated the transfer DNA (T-DNA) by selfing T0 transformants, and among the progeny, they identified mlo1 T-DNA-free mutants, which were regarded as transgene-free $\mathrm{crops}^{87}$. Powdery mildew resistance 4 (PMR4), which encodes a callose synthase, also leads to resistance against $O$. neolycopersici $^{88}$. Another well-known tomato fungal pathosystem is Fusarium oxysporum ${ }^{131}$, which can cause Fusarium wilt disease. The yield of tomato fruit is negligible in highly infected plants. The Solyc08g075770 gene has been identified to function in Fusarium wilt tolerance, and CRISPR-Cas9 knockout transgenic plants exhibited disease susceptibility ${ }^{89}$. Botrytis cinerea is an airborne plant pathogen that causes gray mold disease, resulting in serious economic losses in both pre- and postharvest stages. Tomato is susceptible to postharvest infection by $B$. cinerea $^{133}$. Mitogen-activated protein kinase 3 (MAPK3) has been shown to confer resistance to $B$. cinerea by using CRISPR-Cas9 technology ${ }^{90}$.

Due to undetectable asymptomatic infections and a lack of suitable agricultural chemicals, plant pathogenic bacteria are hard to control, and using genetic resistance against these pathogens is the most efficient strategy ${ }^{130}$. Pseudomonas syringae is the causative agent of the bacterial speck disease in tomato plants, negatively affecting their productivity and marketability. Because Jasmonatezim domain protein 2 (JAZ2) contributes to the defense against $P$. syringae in A. thaliana ${ }^{134}$, researchers used CRISPR-Cas9 to generate tomato dominant JAZ2 repressors lacking the $\mathrm{C}$-terminal jasmonate associated (Jas) domain (JAZ2 $\triangle$ jas). These JAZ2 $\triangle$ jas repressors provide resistance to $P$. syringae, indicating that a CRISPR-Cas9-based strategy for fruit crop protection can be implemented in the field ${ }^{91}$.

\section{Resistance to abiotic stresses}

According to Charles Darwin's evolutionary theory, it is not the most intellectual or strongest species that survives, but the one that is able to adapt to and adjust best to the changing environment in which it finds itself ${ }^{135}$. Abiotic stresses such as drought, flooding, heat, and chilling, especially those under a climate change scenario, pose high risks to species, especially crops ${ }^{136}$. Traditional breeding techniques have greatly increased crop yield, but with the growing demand for food, new approaches are needed to further improve crop production, and CRISPRCas9 technology is the most promising ${ }^{137}$.

Brassinazole resistant 1 (BZR1) regulates brassinosteroid (BR) response and participates in BR-mediated developmental processes. Its ortholog in tomato also controls BR response. BZR1 is also involved in thermotolerance by regulating the Feronia $(F E R)$ genes, as verified by both CRISPR-bzr1- and BZR1-overexpressing lines ${ }^{100}$. Because tomato is a chilling-sensitive crop, its fruit quality is easily damaged due to chilling stress. Li et al. ${ }^{101}$ found that $C$-repeat binding factor 1 (CBF1) protects plants from cold injury, as the $c b f 1$ mutant generated by CRISPR-Cas9 exhibited more severe chilling-injury symptoms with higher electrolyte leakage than WT plants. MAPK3, which participates in resistance against gray mold disease ${ }^{90}$, is also involved in tomato drought response by protecting cell membranes from oxidative damage $^{102}$.

\section{Improvement of tomato fruit quality}

Fruit quality can be defined based on external and internal characteristics. The external quality factors are fruit size, color, and texture, all easily detected with the naked eye. Internal fruit quality attributes, including the levels of nutrients (such as sugar and vitamin) and bioactive compounds (such as lycopene, anthocyanin, and malate), need to be measured by instruments ${ }^{138}$.

In tomato fruit, the number of locules derived from the flower carpels has the greatest effect on tomato fruit size, contributing as much as $50 \%$ to the total variance in fruit enlargement. Locule number is controlled by multiple quantitative trait loci (QTL), a few of which have been identified $^{139}$. Scientists at the Cold Spring Harbor Laboratory used CRISPR-Cas9 technology to rapidly generate larger tomato fruits by destructing the classical CLAVATA-WUSCHEL (CLV-WUS) stem cell circuit ${ }^{140}$. 
Table 2 Current applications of CRISPR-Cas9 in fruit crops

\begin{tabular}{ll}
\hline Crop species & Target genes \\
\hline Resistance to biotic stresses \\
Tomato & CP and Rep of virus \\
Tomato & DCL2 \\
Tomato & DMR6 \\
Tomato & MLO1 \\
Tomato & PMR4 \\
Tomato & SolyC08g075770 \\
Tomato & MAPK3 \\
Tomato & JAZ2 \\
Banana & ORF region of virus \\
Cucumber & elF4E \\
& \\
Grape & MLO7 \\
Grape & WRKY52 \\
Cacao & NPR3 \\
Papaya & alEPIC8 \\
Citrus & LOB1 promoter \\
Apple & DIPM1, 2, 4
\end{tabular}

Resistance to biotic stresses

Resistance to abiotic stresses

$\begin{array}{ll}\text { Tomato } & \text { BZR1 } \\ \text { Tomato } & \text { CBF1 } \\ \text { Tomato } & \text { MAPK3 } \\ \text { Watermelon } & \text { ALS }\end{array}$

Resistance against tomato yellow leaf curl virus 83

Susceptibility to potato virus $X$, tobacco mosaic virus, and tomato $\quad 84,85$ mosaic virus

Resistance against downy mildew $\quad 86$

Resistance against powdery mildew $\quad 87$

Resistance against powdery mildew 88

Susceptibility to Fusarium wilt disease $\quad 89$

Susceptibility to gray mold disease 90

Resistance against bacterial speck disease 91

Resistance against banana streak virus $\quad 92$

Resistance against cucumber vein yellowing virus, zucchini yellow 93 mosaic virus, and papaya ring spot mosaic virus

Resistance against powdery mildew 94

Resistance against gray mold disease 95

Resistance against Phytophthora tropicalis 96

$\begin{array}{ll}\text { Resistance against Phytophthora palmivora } & 97\end{array}$

Resistance against citrus canker $\quad 98,99$

Resistance against fire blight disease $\quad 94$

Decrease in heat stress tolerance $\quad 100$

Decrease in chilling stress tolerance 101

Decrease in drought stress tolerance $\quad 102$

Resistance against herbicide $\quad 103$

Fruits with increasing locule numbers 104

Yellow-colored tomato 105

Pink-colored tomato 106

$\begin{array}{ll}\text { Purple-colored tomato } & 107\end{array}$

Long-shelf life tomato 108

Long-shelf life tomato 109

Repression of genes controlling sugar metabolism 110

Increase in anthocyanin content 107

Increase in GABA content 111

Increase in GABA content 112

Increase in lycopene content 113

Decrease in malate content 114

Production of parthenocarpic fruit $\quad 115$

Production of parthenocarpic fruit 116 
Table 2 continued

\begin{tabular}{|c|c|c|c|}
\hline Crop species & Target genes & Target traits & Refs. \\
\hline Tomato & ARF7 & Production of parthenocarpic fruit & 117 \\
\hline Tomato & MBP21 & Generation of "jointless" fruit stem & 118 \\
\hline Tomato & GAl & Generation of dwarf tomato plants & 119 \\
\hline Tomato & $B O P 1, B O P 2, B O P 3$ & Early flowering with simplified inflorescences & 120 \\
\hline Tomato & $S P, S P 5 G, C L V 3, W U S, G G P 1$ & $\begin{array}{l}\text { Introduction of traits associated with morphology, flower and fruit } \\
\text { production, and ascorbic acid synthesis }\end{array}$ & 121 \\
\hline Tomato & $S P, O V A T E, M U L T, F A S, C Y C B$ & $\begin{array}{l}\text { Introduction of traits associated with morphology, flower number, } \\
\text { tomato size and number, and lycopene synthesis }\end{array}$ & 122 \\
\hline Tomato & SP5G & Generation of loss of day-length-sensitive tomato plants & 123 \\
\hline Cucumber & WIP1 & Generation of gynoecious plant & 124 \\
\hline Groundcherry & $S P, S P 5 G, C L V 1$ & $\begin{array}{l}\text { Introduction of traits associated with morphology, flower } \\
\text { production, and fruit size }\end{array}$ & 125 \\
\hline Kiwifruit & CEN4, CEN & $\begin{array}{l}\text { Generation of a compact plant with rapid terminal flower and fruit } \\
\text { development }\end{array}$ & 126 \\
\hline
\end{tabular}

CRISPR-Cas clustered regularly interspaced short palindromic repeat-CRISPR-associated, ORF open reading frame, GABA $\gamma$-aminobutyric acid

Eight sgRNAs were designed to target the promoter region of the $C L V 3$ gene, and transgenic plants produced more organs and larger fruits than WT plants. The researchers also recreated a known fruit size QTL, locule number $(l c)$ in tomato, generating fruits with increasing locule number ${ }^{104}$. Color and texture are also important aspects of consumer perception of fresh tomatoes ${ }^{141}$. Consumers from different areas have different color preferences. For instance, European and American consumers prefer red tomatoes, while in Asia, pink-colored tomatoes are more popular ${ }^{142,143}$. Researchers have successfully cultivated yellow ${ }^{105}$, pink $^{106}$, and purple ${ }^{107}$ tomatoes by targeting phytoene synthase 1 (PSY1), MYB transcription factor 12 (MYB12), and Anthocyanin 2 (ANT2), respectively. Modifying texture characteristics for a prolonged shelf life has long been a challenge for breeders. The inactivation of ripening inhibitor (RIN) or DNA demethylase 2 (DML2) by CRISPR can lead to incomplete ripening fruits with long shelf life $\mathrm{e}^{144,145}$. However, these fruits usually fail to develop full color, resulting in poor flavor and reduced nutritional value. Hence, obtaining fruits that exhibit good shelf life without affecting other quality aspects is crucial. Two research groups have reported successful harnessing of fruit softening by silencing pectate lyase (PL) and alcobaca $(A L C)$ without reducing tomato organoleptic and nutritional quality ${ }^{108,109}$, suggesting that the CRISPR system might be an excellent tool for fruit crop improvement.

Regarding internal fruit quality, much effort has been made to increase the levels of nutrients and bioactive compounds. Carbohydrates and vitamins are vital nutrients because they provide energy. Several genes are involved in the synthesis and metabolism of sugar and carotenoids (provitamin A carotenoid can be absorbed and converted to vitamin $\mathrm{A}$ in the human body). For example, knocking out mitogen-activated protein kinase 20 (MPK20) disrupted the expression of several genes that control sugar metabolism at both the transcript and protein levels ${ }^{110}$. Bioactive compounds are defined as "extra nutritional constituents that typically occur in small quantities in foods" and usually play roles in the prevention of cardiovascular disease and cancer ${ }^{146}$. Anthocya$\operatorname{nin}^{147}$, malate ${ }^{114}, \gamma$-aminobutyric acid $(\mathrm{GABA})^{111}$, and lycopene $^{113}$ are considered bioactive compounds, and CRISPR-Cas9 technology has been applied to produce anthocyanin-, GABA- and lycopene-enhanced tomato fruits by modulating the expression of key genes in their metabolic pathways ${ }^{107,111-113}$. The key gene that determines tomato malate content, aluminum-activated malate transporter 9 (ALMT9), has also been identified using CRISPR-Cas $9^{114}$.

\section{Domestication of tomato}

Domestication of plants mostly affects the genes controlling plant morphology (seed size, dispersal mechanism, and plant architecture) and physiology (timing of germination, flowering, and ripening) ${ }^{148,149}$. To achieve the ideotype, classical breeding or modern "rewilding" crop breeding have introduced alleles from wild relatives into cultivated species. However, these techniques are time-consuming. An alternative strategy is direct manipulation of wild crops at the gene level to domesticate them de novo and harness their adaptation to adverse 
environments ${ }^{150}$. This de novo domestication has been substantially accelerated by the CRISPR-Cas9 technology.

Parthenocarpy, a fertilization-independent seedless fruit development, is regarded as a desirable agronomic trait in fruit crops: (i) it is advantageous for stable crop yield in fluctuating environments; (ii) it saves energy when separating the seeds from processed products for industrial purposes; and (iii) consumers prefer seedless over seeded fruits ${ }^{115-117}$. Klap et al. ${ }^{15}$ confirmed that a mutation in agamous-like 6 (AGL6) is responsible for parthenocarpic fruit production under heat stress conditions; because the mutant is of normal weight and shape, without homeotic changes, AGL6 is an attractive gene for parthenocarpy. Elevated gibberellin or auxin signaling can induce parthenocarpy without fertilization. The mutants produced by the knock out of indole-3-acetic acid inducible 9 (IAA9) and auxin response factor 7 (ARF7), both involved in the auxin signaling pathway, produced seedless fruits, which is a characteristic of parthenocarpic tomato $^{116,117}$. The joint is a weak region of the stem that allows the fruit to drop from the plant. Wild species benefit from dropping fruit because this process contributes to seed dispersal, but because they use picking manipulators, farmers prefer to have fruit hanging on the plant. Breeders have been trying to obtain a mutant that eliminates the flower abscission zone (by which unfertilized flowers or ripe fruit are shed from the plant) and provides a "jointless" fruit stem ${ }^{151,152}$. Roldan et al. ${ }^{118}$ developed the MADS-box protein 21 (MBP21) loss-offunction mutant $m b p 21$ exhibiting the jointless phenotype using CRISPR-Cas9 technology ${ }^{118}$. Fruits are easier to pick, and nutrients are transported over shorter distances from the roots to the leaves in dwarf plants compared with normal plants. Dwarf plants are also more likely to survive when exposed to strong winds. Heritable dwarf tomato plants have been generated by inactivating the gibberellic-acid insensitive (GAI) gene, and these plants can be useful in windy environments. However, the reduced fruit weight and seed number issues of these dwarf mutants need to be solved first ${ }^{119}$. Plant productivity depends on flowers, and inflorescence architecture determines flower production. CRISPR-Cas9 technology was used to silence the tomato blade-onpetiole $(B O P)$ gene to test whether it has the same function as its homolog in A. thaliana (leaf complexity and organ abscission), which affects inflorescence architecture. Notably, the CRISPR-bop1/2/3 triple mutant flowered faster than the WT, but with extremely simplified inflorescences ${ }^{120}$.

Domestication of wild tomato species for commercial cultivation usually requires numerous phenotypes to be changed, including fruit setting and size, ripening synchrony, flowering and day-length sensitivity, and nutrient content ${ }^{121}$. Two research groups have recently devised a
CRISPR-Cas9 technology that combines agronomically desirable traits with useful traits present in wild lines. One group targeted six loci of five genes critical for the productivity of present tomato lines, enabling the de novo domestication of wild Solanum pimpinellifolium whose morphology was altered, together with the size, number, and nutritional value of its fruits ${ }^{122}$. The other group introduced desirable traits into $S$. pimpinellifolium by editing coding sequences, cis-regulatory regions, or upstream ORFs of genes associated with morphology, flower and fruit production, and ascorbic acid synthesis ${ }^{121}$.

Sensitivity to day-length limits the geographical distribution of crops. Therefore, modification of the photoperiod response can help accelerate crop domestication processes. The loss of the day-length-sensitive tomato mutant produced by knocking out self-pruning 5G (SP5G) showed a quick burst of flower production that translated into an early fruit yield ${ }^{123}$.

\section{Current applications of CRISPR-Cas9 in other fruit crops}

The use of CRISPR-Cas9 technology is not limited to tomato. It has also been successfully applied to several other fruit crops, including strawberry ${ }^{153}$, banana ${ }^{154}$, grape ${ }^{155}$, apple $^{156}$, watermelon ${ }^{157}$, and kiwifruit ${ }^{158}$. As a model organism, strawberry is often used for the functional analysis of specific genes. For instance, targeting $R 2 R 3 M Y B$ transcription factor 10 (MYB10) leads to the generation of loss-of-coloration fruits ${ }^{159,160}$. Zhou et al. ${ }^{153}$ used CRISPRCas9 to target auxin response factor 8 (ARF8) and identified that arf 8 homozygous mutants show faster seedling growth than WT plants. The tomato MADS-box gene 6 (TM6) is reported to play a predominant role in stamen development ${ }^{161}$. To characterize its function in strawberry, the CRISPR-Cas9 system was applied to an octoploid species, and the phenotypic analysis of tm6 mutants revealed severe defects in their anthers, indicating that TM6 played an essential role in flower development ${ }^{162}$. In addition, the CRISPR-Cas9 strategy was used to investigate the biological role of YUCCA 10 (YUC10) in auxin synthesis during strawberry fruit development. When YUC10 was knocked out, a significant reduction in free auxin was observed in yuc10 mutants ${ }^{163}$. In addition to the functional study in strawberry, an increasing number of researchers are focusing on CRISPR-Cas9-mediated genome editing for improvement of other fruit crops. Here, we summarize the recent applications of CRISPR-Cas9 to other fruit crops considering the following aspects: resistance to biotic stresses, resistance to abiotic stresses, and domestication of fruit crops (Table 2).

\section{Resistance to biotic stresses}

In tropical and subtropical countries, the banana streak virus is a major challenge in banana breeding ${ }^{92}$. As mentioned above, one strategy for improving resistance to 
viruses is targeting their genomes with CRISPR-Cas9. Tripathi et al. ${ }^{92}$ used this system to inactivate the endogenous banana streak virus and found that $75 \%$ of the edited plants remained asymptomatic in comparison to the non-edited control. Plant RNA viruses require a host factor, such as the eukaryotic translation initiation factor $4 \mathrm{E}$ (eIF4E), to maintain their life cycle. If the factor is inactivated, viral infectivity is disrupted. A virus-resistant cucumber mutant was developed using CRISPR-Cas9 to disrupt the function of eIF4E. As expected, the eif4e mutant exhibited immunity to cucumber vein yellowing virus, zucchini yellow mosaic virus, and papaya ring spot mosaic virus ${ }^{93}$. Fungal diseases can cause drastic losses in grapevine yield and grape berry quality. Two genes, mildew resistance locus $O 7$ (MLO7) and WRKY transcription factor 52 (WRKY52), are known to be involved in Erysiphe necator and $B$. cinerea resistance, respectively. Two research groups validated the functions of these genes using CRISPR-Cas9. Both loss-of-function mutants showed increased immunity ${ }^{94,95}$. This technology was also used in cacao and papaya to increase resistance against Phytophthora tropicalis and Phytophthora palmivora ${ }^{96,97}$. Citrus canker, caused by Xanthomonas citri, is a severe disease among most commercial citrus cultivars and is responsible for substantial economic losses worldwide. Two recent publications ${ }^{98,99}$ have reported the use of CRISPR-Cas9 for generating citrus plants resistant to citrus canker by targeting the promoter region of the lateral organ boundaries 1 ( $L O B 1$ ) gene in citrus; the mutated lines showed high degrees of resistance to $X$. citri infection. Similarly, in apple protoplasts, the genes encoding DspA/E-interacting proteins (DIPM1, DIPM2, and DIPM4) were knocked out to improve resistance against Erwinia amylovora ${ }^{94}$. Date palm is an important fruit crop in desert agriculture. Due to its large and complex genome and high frequency of single-nucleotide polymorphisms, the application of CRISPR-Cas9 is a challenging task, and therefore, few genetic improvement studies have been performed. However, Satter et al. ${ }^{164}$ presented a generalized stepwise and basic strategy for the theoretical implications of CRISPR-Cas9, addressing its potential applications in date palm.

\section{Resistance to abiotic stresses}

Field watermelons are severely threatened by weeds, but the use of herbicides also affects their growth. Therefore, herbicide-resistant watermelons should be obtained, which is difficult to achieve via traditional breeding. In recent years, CRISPR-mediated single-nucleotide conversion has been used to develop herbicide-resistant rice $^{56}$. To introduce this new base-editing system in watermelon, Tian et al. ${ }^{103}$ selected acetolactate synthase $(A L S)$, a gene in which point mutations confer a high level of herbicide resistance. The transgene-free als mutants and WT plants were treated with the herbicide tribenuron, and while all WT plants were severely damaged, the als mutants were not, suggesting the successful establishment of a CRISPR base-editing system and herbicide-resistant watermelons ${ }^{103}$.

\section{Domestication of fruit crops}

Gynoecious lines benefit cucumber breeding, as they allow earlier generation of hybrids, higher yield, and more concentrated fruit set; eliminate the requirement for artificial emasculation; and reduce the labor cost of crossing compared to monoecious lines. WIP domaincontaining protein 1 (WIP1) inhibits carpel development in cucumber, and the loss-of-function wip1 mutant displays a gynoecious phenotype, bearing only female flowers in upper nodes ${ }^{124}$. Lemmon et al. ${ }^{125}$ domesticated an orphan crop, groundcherry, a wild Solanaceae grown in Central and South America. Using CRISPR-Cas9, three orthologs of tomato (self-pruning (SP), SP5G, and CLV1) that control plant architecture, flower production, and fruit size, respectively, were introduced into groundcherry, thereby improving these major productive characters in this crop. This successful application will accelerate the domestication of orphan crops by introducing known agronomic traits from distantly related model crops ${ }^{125}$. Kiwifruit is a recently domesticated fruit crop with large potential for improvement. By inactivating centroradialis 4 (CEN4) and CEN, which have been validated as repressors of flowering, the original climbing woody perennial was transformed into a compact plant with rapid terminal flower and fruit development ${ }^{126}$.

\section{Concluding remarks}

CRISPR-Cas9 technology has revolutionized crop breeding since its first application in 2013. The major breakthroughs were the generation of disease-resistant and environment-adaptive fruit crops, as well as improvement of fruit quality. Notably, the DNA-free delivery of preassembled CRISPR-Cas9 ribonucleoproteins has been conducted in plant protoplasts of $A$. thaliana, rice, tobacco, lettuce, wheat, and potato ${ }^{165-168}$. Plants originating from this technology might be considered non-GM crops. This characterization would open the door for the development of fruit crops with superior phenotypes and allow their commercialization and marketing even in countries where GM crops are unacceptable $^{169}$. In April 2016, the FDA indicated that the CRISPR-edited mushroom could enter the market without oversight, making it the first CRISPR-edited organism to receive such authorization from the US government ${ }^{16,170}$. In 2017, the FDA allowed the marketing of false flax, with enhanced omega-3 oil, and droughttolerant soybean, clearly indicating that CRISPR-edited plants can be cultivated and sold free from regulation ${ }^{17}$ 
and thereby providing great confidence to research focusing on the application of CRISPR to fruit crops.

However, the growth of CRISPR-edited crops faces sociopolitical challenges, including public acceptance and government regulation ${ }^{171}$. Although transgene-free organisms edited by CRISPR-Cas9 are not currently regulated in the US, whether to govern the use of CRISPR technologies is still being discussed in China and Japan ${ }^{172}$. According to the decision of Europe's highest court earlier in 2018, gene-edited crops should be subject to the same stringent regulations that govern conventional GM organisms, which is a major setback for proponents, including many scientists ${ }^{173}$. With further advances in CRISPR technology and the establishment of an evaluation system, more countries might be willing to foster an optimistic and inclusive attitude toward CRISPR-edited crops. As researchers, in addition to further investigating CRISPR technology to ensure maximum benefit while minimizing risks, we need to be concerned with public acceptance. Most importantly, the basic aspects of this technology need to be explained sufficiently well to facilitate rational public discourse, increasing public confidence in the safety and advantages of CRISPR-edited crops. Governments might then express a laissez faire attitude after gaining strong public trust.

\section{Acknowledgements}

This work was supported by grants from National Key R\&D Program of China (2016YFD0400901) and National Natural Sciences Foundation of China

(31622050 and 31672208) to H.Z. T.W. was supported by a fellowship from the Chinese Scholarship Council (201706350174).

\section{Author details \\ ${ }^{1}$ College of Food Science and Nutritional Engineering, China Agricultural University, 100083 Beijing, China. ${ }^{2}$ Key Laboratory of Food Nutrition and Safety of Shandong Normal University, College of Life Science, Shandong Normal University, 250014 Jinan, China. ${ }^{3}$ College of Food Science and Nutritional Engineering, China Agricultural University, 100083 Beijing, China}

\section{Author contributions}

T.W. and H.Z. planned the manuscript outline. T.W. wrote the draft and created the figures and tables. H.Z. and H.Z. revised and proofread the manuscript. All authors read and approved the final manuscript.

\section{Conflict of interest}

The authors declare that they have no conflict of interest.

Received: 26 November 2018 Revised: 25 February 2019 Accepted: 26 April 2019

Published online: 15 June 2019

\section{References}

1. Giovannoni, J., Nguyen, C., Ampofo, B., Zhong, S. \& Fei, Z. The epigenome and transcriptional dynamics of fruit ripening. Annu. Rev. Plant Biol. 68, 61-84 (2017).

2. Ramdath, D. D., Isaacs, R. L., Teelucksingh, S. \& Wolever, T. M. Glycaemic index of selected staples commonly eaten in the Caribbean and the effects of boiling v. crushing. Br. J. Nutr. 91, 971-977 (2004).
3. Vayalil, P. K. Date fruits (Phoenix dactylifera Linn): an emerging medicinal food Crit. Rev. Food Sci. 52, 249-271 (2012).

4. Singh, B., Singh, J. P., Kaur, A. \& Singh, N. Bioactive compounds in banana and their associated health benefits - a review. Food Chem. 206, 1-11 (2016).

5. Karkute, S. G., Singh, A. K., Gupta, O. P., Singh, P. M. \& Singh, B. CRISPR/Cas9 mediated genome engineering for improvement of horticultural Crops. Front. Plant Sci. 8, 01635 (2017).

6. Hickey, J. M. et al. Genomic prediction unifies animal and plant breeding programs to form platforms for biological discovery. Nat. Genet. 49, 1297-1303 (2017).

7. Meyer, R. S. \& Purugganan, M. D. Evolution of crop species: genetics of domestication and diversification. Nat. Rev. Genet. 14, 840-852 (2013).

8. Tester, M. \& Langridge, P. Breeding technologies to increase crop production in a Changing world. Science 327, 818-822 (2010).

9. Bigliardi, B. \& Galati, F. Innovation trends in the food industry: the case of functional foods. Trends Food Sci. Technol. 31, 118-129 (2013).

10. Parmar, N. et al. Genetic engineering strategies for biotic and abiotic stress tolerance and quality enhancement in horticultural crops: a comprehensive review. 3 Biotech 7, 239 (2017).

11. Bawa, A. S. \& Anilakumar, K. R. Genetically modified foods: safety, risks and public concerns-a review. J. Food Sci. Technol. 50, 1035-1046 (2013).

12. Gould, F. et al. in Genetically Engineered Crops: Experiences and Prospects (eds Board on Agriculture and Natural Resources) Ch. 6 (National Academies Press, Washington, DC, 2016).

13. Millstone, E., Stirling, A. \& Glover, D. Regulating genetic engineering the limits and politics of knowledge. Issues Sci. Technol. 31, 23-26 (2015).

14. Falck-Zepeda, J. et al. Estimates and implications of the costs of compliance with biosafety regulations in developing countries. GM Crops Food 3, 52-59 (2012).

15. Jefferson, D. J., Graff, G. D., Chi-Ham, C. L. \& Bennett, A. B. The emergence of agbiogenerics. Nat. Biotechnol. 33, 819-823 (2015).

16. Kim, J. \& Kim, J. Bypassing GMO regulations with CRISPR gene editing. Nat. Biotechnol. 34, 1014-1015 (2016).

17. Waltz, E. With a free pass, CRISPR-edited plants reach market in record time. Nat. Biotechnol. 36, 6-7 (2018).

18. Zaidi, S. S., Mukhtar, M. S. \& Mansoor, S. Genome editing: targeting susceptibility genes for plant disease resistance. Trends Biotechnol. 36, 898-906 (2018).

19. Sternberg, S. H., Richter, H., Charpentier, E. \& Qimron, U. Adaptation in CRISPRCas systems. Mol. Cell 61, 797-808 (2016).

20. Ishino, Y., Shinagawa, H., Makino, K., Amemura, M. \& Nakata, A. Nucleotide sequence of the iap gene, responsible for alkaline phosphatase isozyme conversion in Escherichia coli, and identification of the gene product. J. Bacteriol. 169, 5429-5433 (1987).

21. Jansen, R., Embden, J. D. A. V., Gaastra, W. \& Schouls, L. M. Identification of genes that are associated with DNA repeats in prokaryotes. Mol. Microbiol. 43, 1565-1575 (2002).

22. Bolotin, A., Ouinquis, B., Sorokin, A. \& Ehrlich, S. D. Clustered regularly interspaced short palindrome repeats (CRISPRs) have spacers of extrachromosomal origin. Microbiology 151, 2551-2561 (2005).

23. Mojica, F. J. M., Díez-Villaseñor, C. S., García-Martínez, J. S. \& Soria, E. Intervening sequences of regularly spaced prokaryotic repeats derive from foreign genetic elements. J. Mol. Evol. 60, 174-182 (2005).

24. Pourcel, C., Salvignol, G. \& Vergnaud, G. CRISPR elements in Yersinia pestis acquire new repeats by preferential uptake of bacteriophage DNA, and provide additional tools for evolutionary studies. Microbiology 151, 653-663 (2005).

25. Shan, Q. et al. Targeted genome modification of crop plants using a CRISPRCas system. Nat. Biotechnol. 31, 686-688 (2013).

26. Cong, L. et al. Multiplex genome engineering using CRISPR/Cas systems. Science 339, 819-823 (2013).

27. Kaboli, S. \& Babazada, H. CRISPR mediated genome engineering and its application in industry. Curr. Issues Mol. Biol. 26, 81-92 (2018).

28. Hille, F. \& Charpentier, E. CRISPR-Cas: biology, mechanisms and relevance. Philos. Trans. R. Soc. Ser. B 371, 20150496 (2016).

29. Nam, K. H. et al. Cas5d protein processes pre-crRNA and assembles into a cascade-like interference complex in subtype I-C/Dvulg CRISPR-Cas system. Structure 20, 1574-1584 (2012).

30. Rouillon, C. et al. Structure of the CRISPR interference complex CSM reveals key similarities with cascade. Mol. Cell 52, 124-134 (2013). 
31. Gasiunas, G., Barrangou, R., Horvath, P. \& Siksnys, V. Cas9-crRNA ribonucleoprotein complex mediates specific DNA cleavage for adaptive immunity in bacteria. Proc. Natl. Acad. Sci. USA 109, E2579-E2586 (2012).

32. Doudna, J. A. \& Charpentier, E. The new frontier of genome engineering with CRISPR-Cas9. Science 346, 1258096 (2014).

33. Amitai, G. \& Sorek, R. CRISPR-Cas adaptation: insights into the mechanism of action. Nat. Rev. Microbiol. 14, 67-76 (2016).

34. Wang, H., La Russa, M. \& Qi, L. S. CRISPR/Cas9 in genome editing and beyond. Annu. Rev. Biochem. 85, 227-264 (2016).

35. Yin, K., Gao, C. \& Qiu, J. Progress and prospects in plant genome editing. Nat. Plants 3, 17107 (2017).

36. Marraffini, L. A. in Streptococcus pyogenes: Basic Biology to Clinical Manifestations [Internet] (eds Ferretti, J. J., Stevens, D. L. \& Fischetti, V. A.) Ch. 11 (University of Oklahoma Health Sciences Center, Oklahoma City, 2016).

37. Liu, X., Xie, C., Si, H. \& Yang, J. CRISPR/Cas9-mediated genome editing in plants. Methods 121-122, 94-102 (2017).

38. Donohoue, P. D., Barrangou, R. \& May, A. P. Advances in industrial biotechnology using CRISPR-Cas systems. Trends Biotechnol. 36, 134-146 (2018).

39. Liang, W., Wersch, S., Tong, M. \& Li, X. TIR-NB-LRR immune receptor SOC3 pairs with truncated TIR-NB protein CHS1 or TN2 to monitor the homeostasis of E3 ligase SAUL1. New Phytol. 221, 2054-2066 (2018).

40. Mao, Y. et al. Manipulating plant RNA-silencing pathways to improve the gene editing efficiency of CRISPR/Cas9 systems. Genome Biol. 19, 149 (2018).

41. Li, R., Fu, D., Zhu, B., Luo, Y. \& Zhu, H. CRISPR/Cas9-mediated mutagenesis of IncRNA1459 alters tomato fruit ripening. Plant J. 94, 513-524 (2018).

42. Chang, $\mathrm{H}$. et al. CRISPR/cas9, a novel genomic tool to knock down microRNA in vitro and in vivo. Sci. Rep. 6, 22312 (2016).

43. Seth, K. \& Harish Current status of potential applications of repurposed Cas 9 for structural and functional genomics of plants. Biochem. Biophys. Res. Commun. 480, 499-507 (2016).

44. Hess, G. T., Tycko, J., Yao, D. \& Bassik, M. C. Methods and applications of CRISPR-mediated base editing in eukaryotic genomes. Mol. Cell 68, 26-43 (2017).

45. Steinert, J., Schiml, S., Fauser, F. \& Puchta, H. Highly efficient heritable plant genome engineering using Cas9 orthologues from Streptococcus thermophilus and Staphylococcus aureus. Plant J. 84, 1295-1305 (2015).

46. Kaya, H., Mikami, M., Endo, A., Endo, M. \& Toki, S. Highly specific targeted mutagenesis in plants using Staphylococcus aureus Cas9. Sci. Rep. 6, 26871 (2016).

47. Hu, X. et al. Expanding the range of CRISPR/Cas9 genome editing in rice. Mol. Plant 9, 943-945 (2016)

48. Lee, K. et al. Activities and specificities of CRISPR/Cas9 and Cas12a nucleases for targeted mutagenesis in maize. Plant Biotechnol. J. 17, 362-372 (2019).

49. Tang, $X$. et al. A CRISPR-Cpf1 system for efficient genome editing and transcriptional repression in plants. Nat. Plants 3, 17018 (2017).

50. Kim, H. et al. CRISPR/Cpf1-mediated DNA-free plant genome editing. Nat Commun. 8, 14406 (2017)

51. Endo, A., Masafumi, M., Kaya, H. \& Toki, S. Efficient targeted mutagenesis of rice and tobacco genomes using Cpf1 from Francisella novicida. Sci. Rep. 6 38169 (2016)

52. Aman, R. et al. RNA virus interference via CRISPR/Cas13a system in plants. Genome Biol. 19, 1 (2018).

53. Abudayyeh, O. O. et al. RNA targeting with CRISPR-Cas13. Nature $\mathbf{5 5 0}$ 280-284 (2017).

54. Mikami, M., Toki, S. \& Endo, M. Precision targeted mutagenesis via Cas9 paired nickases in rice. Plant Cell Physiol. 57, 1058-1068 (2016).

55. Fauser, F., Schiml, S. \& Puchta, H. Both CRISPR/Cas-based nucleases and nickases can be used efficiently for genome engineering in Arabidopsis thaliana. Plant J. 79, 348-359 (2014).

56. Shimatani, Z. et al. Targeted base editing in rice and tomato using a CRISPRCas9 cytidine deaminase fusion. Nat. Biotechnol. 35, 441-443 (2017).

57. Zong, Y. et al. Precise base editing in rice, wheat and maize with a Cas9cytidine deaminase fusion. Nat. Biotechnol. 35, 438-440 (2017).

58. Zhang, F. et al. EIN2 mediates direct regulation of histone acetylation in the ethylene response. Proc. Natl. Acad. Sci. USA 114, 10274-10279 (2017).

59. Piatek, A. et al. RNA-guided transcriptional regulationin planta via synthetic dCas9-based transcription factors. Plant Biotechnol. J. 13, 578-589 (2015).
60. Zhang, F., LeBlanc, C., Irish, V. F. \& Jacob, Y. Rapid and efficient CRISPR/Cas9 gene editing in citrus using the YAO promoter. Plant Cell Rep. 36, 1883-1887 (2017).

61. Yan, L. et al. High-efficiency genome editing in Arabidopsis using YAO promoter-driven CRISPR/Cas9 system. Mol. Plant 8, 1820-1823 (2015).

62. Mao, Y. et al. Development of germ-line-specific CRISPR-Cas9 systems to improve the production of heritable gene modifications in Arabidopsis. Plant Biotechnol. J. 14, 519-532 (2016).

63. Wolter, F., Klemm, J. \& Puchta, H. Efficientin planta gene targeting in Arabidopsis using egg cell-specific expression of the Cas9 nuclease of Staphylococcus aureus. Plant J. 94, 735-746 (2018).

64. Wang, Z. et al. Egg cell-specific promoter-controlled CRISPR/Cas9 efficiently generates homozygous mutants for multiple target genes in Arabidopsis in a single generation. Genome Biol. 16, 144 (2015).

65. Hyun, Y. et al. Site-directed mutagenesis in Arabidopsis thaliana using dividing tissue-targeted RGEN of the CRISPR/Cas system to generate heritable null alleles. Planta 241, 271-284 (2015).

66. Osakabe, Y. et al. Optimization of CRISPR/Cas9 genome editing to modify abiotic stress responses in plants. Sci. Rep. 6, 26685 (2016).

67. Eid, A., Ali, Z. \& Mahfouz, M. M. High efficiency of targeted mutagenesis in Arabidopsis via meiotic promoter-driven expression of Cas9 endonuclease. Plant Cell Rep. 35, 1555-1558 (2016).

68. Xu, P., Su, H., Chen, W. \& Lu, P. The application of a meiocyte-specific CRISPR/ Cas9 (MSC) system and a suicide-MSC system in generating inheritable and stable mutations in Arabidopsis. Front. Plant Sci. 9, 01007 (2018).

69. Feng, $C$. et al. High-efficiency genome editing using admc1 promotercontrolled CRISPR/Cas9 system in maize. Plant Biotechnol. J. 16, 1848-1857 (2018)

70. Tsutsui, H. \& Higashiyama, T. pKAMA-ITACHI vectors for highly efficient CRISPR/Cas9-mediated gene knockout in Arabidopsis thaliana. Plant Cell Physiol. 58, w191 (2017).

71. Yu, T. et al. Genome-wide identification of long non-coding RNA targets of the tomato MADS box transcription factor RIN and function analysis. Ann Bot. 123, 469-482 (2019)

72. Char, S. N. et al. An Agrobacterium-delivered CRISPR/Cas9 system for highfrequency targeted mutagenesis in maize. Plant Biotechnol. J. 15, 257-268 (2017)

73. Ma, X. et al. A robust CRISPR/Cas9 system for convenient, high-efficiency multiplex genome editing in monocot and dicot plants. Mol. Plant $\mathbf{8}$ 1274-1284 (2015)

74. Fan, D. et al. Efficient CRISPR/Cas9-mediated targeted mutagenesis in populus in the first generation. Sci. Rep. 5, 12217 (2015)

75. Gao, J. et al. CRISPR/Cas9-mediated targeted mutagenesis in Nicotiana tabacum. Plant Mol. Biol. 87, 99-110 (2015).

76. Hashimoto, R., Ueta, R., Abe, C., Osakabe, Y. \& Osakabe, K. Efficient multiplex genome editing induces precise, and self-ligated type mutations in tomato plants. Front. Plant Sci. 9, 00916 (2018).

77. Nakayasu, M. et al. Generation of a-solanine-free hairy roots of potato by CRISPR/Cas9 mediated genome editing of the St16DOX gene. Plant Physiol. Biochem. 131, 70-77 (2018).

78. Li, C. et al. Expanded base editing in rice and wheat using a Cas9-adenosine deaminase fusion. Genome Biol. 19, 59 (2018).

79. Oi, W. et al. High-efficiency CRISPR/Cas9 multiplex gene editing using the glycine tRNA-processing system-based strategy in maize. BMC Biotechnol. 16 58 (2016).

80. Xie, K., Minkenberg, B. \& Yang, Y. Boosting CRISPR/Cas9 multiplex editing capability with the endogenous tRNA-processing system. Proc. Natl. Acad. Sci. USA 112, 3570-3575 (2015)

81. Rieger, M. in Introduction to Fruit Crops (ed. Rieger, M.) Ch. 1 (Food Products Press, Boca Raton, 2006).

82. Miller, A. J. \& Gross, B. L. From forest to field: perennial fruit crop domestication. Am. J. Bot. 98, 1389-1414 (2011).

83. Tashkandi, M., Ali, Z., Aljedaani, F., Shami, A. \& Mahfouz, M. M. Engineering resistance against Tomato yellow leaf curl virus via the CRISPR/Cas9 system in tomato. Plant Signal. Behav. 13, e1525996 (2018).

84. Wang, Z. et al. A novel DCL2-dependent miRNA pathway in tomato affects susceptibility to RNA viruses. Gene. Dev. 32, 1155-1160 (2018).

85. Wang, $\mathrm{T}$. et al. Tomato DCL2b is required for the biosynthesis of 22-nt small RNAs, the resulting secondary siRNAs, and the host defense against ToMV. Hortic. Res. 5, 62 (2018). 
86. Thomazella, D., Brail, Q., Dahlbeck, D. \& Staskawicz, B. CRISPR-Cas9 mediated mutagenesis of a DMR6 ortholog in tomato confers broad-spectrum disease resistance. BioRxiv https://doi.org/10.1101/064824 (2016).

87. Nekrasov, $V$. et al. Rapid generation of a transgene-free powdery mildew resistant tomato by genome deletion. Sci. Rep. 7, 482-486 (2017).

88. Koseoglou, E. Wageningen University, http://edepot.wur.nl/422311 (2017).

89. Prihatna, C., Barbetti, M. J. \& Barker, S. J. A novel tomato Fusarium Wilt tolerance gene. Front. Microbiol. 9, 1226 (2018)

90. Zhang, S. et al. Knockout of SIMAPK3 reduced disease resistance to Botrytis cinerea in tomato plants. J. Agric. Food Chem. 66, 8949-8956 (2018).

91. Ortigosa, A., Gimenez-lbanez, S., Leonhardt, N. \& Solano, R. Design of a bacterial speck resistant tomato by CRISPR/Cas9-mediated editing ofSIJAZ2. Plant Biotechnol. J. 17, 665-673 (2019).

92. Tripathi, J. N. et al. CRISPR/Cas9 editing of endogenous banana streak virus in the B genome of Musa spp. overcomes a major challenge in banana breeding. Commun. Biol. 2, 46 (2019).

93. Chandrasekaran, J. et al. Development of broad virus resistance in nontransgenic cucumber using CRISPR/Cas9 technology. Mol. Plant Pathol. 17, 1140-1153 (2016).

94. Malnoy, M. et al. DNA-free genetically edited grapevine and apple protoplast using CRISPR/Cas9 ribonucleoproteins. Front Plant Sci. 7, 1904 (2016).

95. Wang, $X$. et al. CRISPR/Cas9-mediated efficient targeted mutagenesis in grape in the first generation. Plant Biotechnol. J. 16, 844-855 (2018).

96. Fister, A. S., Landherr, L., Maximova, S. N. \& Guiltinan, M. J. Transient expression of CRISPR/Cas9 machinery targeting TCNPR3 enhances defense response in Theobroma cacao. Front Plant Sci. 9, 268 (2018).

97. Gumtow, R., Wu, D., Uchida, J. \& Tian, M. A Phytophthora palmivora extracellular cystatin-like protease inhibitor targets papain to contribute to virulence on papaya. Mol. Plant Microbe. 31, 363-373 (2018).

98. Jia, H., Orbovic, V., Jones, J. B. \& Wang, N. Modification of the PthA4 effector binding elements in type I CsLOB1 promoter using Cas9/sgRNA to produce transgenic Duncan grapefruit alleviating Xcc $\triangle$ pthA4:dCsLOB1.3 infection. Plant Biotechnol. J. 14, 1291-1301 (2016).

99. Peng, A. et al. Engineering canker-resistant plants through CRISPR/Cas9targeted editing of the susceptibility gene CSLOB1 promoter in citrus. Plant Biotechnol. J. 15, 1509-1519 (2017).

100. Yin, Y. et al. BZR1 transcription factor regulates heat stress tolerance through FERONIA receptor-like kinase-mediated reactive oxygen species signaling in tomato. Plant Cell Physiol. 59, 2239-2254 (2018).

101. Li, R. et al. Reduction of tomato-plant chilling tolerance by CRISPR-Cas9mediated SICBF1 mutagenesis. J. Agric. Food Chem. 66, 9042-9051 (2018).

102. Wang, L. et al. Reduced drought tolerance by CRISPR/Cas9-mediated SIMAPK3 mutagenesis in tomato plants. J. Agric. Food Chem. 65, 8674-8682 (2017).

103. Tian, S. et al. Engineering herbicide-resistant watermelon variety through CRISPR/Cas9-mediated base-editing. Plant Cell Rep. 37, 1353-1356 (2018).

104. Rodríguez-Leal, D., Lemmon, Z. H., Man, J., Bartlett, M. E. \& Lippman, Z. B. Engineering quantitative trait variation for crop improvement by genome editing. Cell 171, 470-480 (2017).

105. Filler Hayut, S., Melamed Bessudo, C. \& Levy, A. A. Targeted recombination between homologous chromosomes for precise breeding in tomato. Nat Commun. 8, 15605 (2017)

106. Deng, L. et al. Efficient generation of pink-fruited tomatoes using CRISPR/ Cas9 system. J. Genet. Genom. 45, 51-54 (2018).

107. Čermák, T., Baltes, N. J., Čegan, R., Zhang, Y. \& Voytas, D. F. High-frequency, precise modification of the tomato genome. Genome Biol. 16, 232 (2015).

108. Uluisik, S. et al. Erratum: Corrigendum: genetic improvement of tomato by targeted control of fruit softening. Nat. Biotechnol. 34, 1072 (2016).

109. Yu, Q. et al. CRISPR/Cas9-induced targeted mutagenesis and gene replacement to generate long-shelf life tomato lines. Sci. Rep. 7, 11874 (2017).

110. Chen, L. et al. Evidence for a specific and critical role of mitogen-activated protein kinase 20 in uni-to-binucleate transition of microgametogenesis in tomato. New Phytol. 219, 176-194 (2018).

111. Nonaka, S., Arai, C., Takayama, M., Matsukura, C. \& Ezura, H. Efficient increase of $\mathrm{\gamma}$-aminobutyric acid (GABA) content in tomato fruits by targeted mutagenesis. Sci. Rep. 7, 7057 (2017)

112. Li, R. et al. Multiplexed CRISPR/Cas9-mediated metabolic engineering of $\gamma$ aminobutyric acid levels in Solanum lycopersicum. Plant Biotechnol. J. 16, 415-427 (2018)

113. Li, X. et al. Lycopene is enriched in tomato fruit by CRISPR/Cas9-mediated multiplex genome editing. Front. Plant Sci. 9, 559 (2018).
114. Ye, J. et al. An inDel in the promoter of Al-activated malate transporter9 selected during tomato domestication determines fruit malate contents and Aluminum tolerance. Plant Cell 29, 2249-2268 (2017).

115. Klap, C. et al. Tomato facultative parthenocarpy results from SIAGAMOUS-LIKE 6 loss of function. Plant Biotechnol. J. 15, 634-647 (2017).

116. Ueta, R. et al. Rapid breeding of parthenocarpic tomato plants using CRISPR Cas9. Sci. Rep. 7, 507 (2017).

117. Hu, J., Israeli, A., Ori, N. \& Sun, T. The interaction between DELLA and ARF/IAA mediates crosstalk between gibberellin and auxin signaling to control fruit initiation in tomato. Plant Cell 30, 1710-1728 (2018).

118. Roldan, M. V. G. et al. Natural and induced loss of function mutations in SIMBP21 MADS-box gene led to jointless-2 phenotype in tomato. Sci. Rep. 7 4402 (2017)

119. Tomlinson, L. et al. Using CRISPR/Cas9 genome editing in tomato to create a gibberellin-responsive dominant dwarf DELLA allele. Plant Biotechnol. J. 17, 132-140 (2019).

120. Xu, C., Park, S. J., Van Eck, J. \& Lippman, Z. B. Control of inflorescence architecture in tomato by BTB/POZ transcriptional regulators. Gene Dev. $\mathbf{3 0}$ 2048-2061 (2016).

121. Li, T. et al. Domestication of wild tomato is accelerated by genome editing Nat. Biotechnol. 36, 1160-1163 (2018).

122. Zsögön, A. et al. De novo domestication of wild tomato using genome editing. Nat. Biotechnol. 36, 1211-1216 (2018).

123. Soyk, S. et al. Variation in the flowering gene SELF PRUNING 5G promotes day-neutrality and early yield in tomato. Nat. Genet. 49, 162-168 (2017).

124. $\mathrm{Hu}, \mathrm{B}$. et al. Engineering non-transgenic gynoecious cucumber using an improved transformation protocol and optimized CRISPR/Cas9 system. Mol. Plant 10, 1575-1578 (2017).

125. Lemmon, Z. H. et al. Rapid improvement of domestication traits in an orphan crop by genome editing. Nat. Plants 4, 766-770 (2018).

126. Varkonyi-Gasic, E. et al. Mutagenesis of kiwifruit CENTRORADIALIS-like genes transforms a climbing woody perennial with long juvenility and axillary flowering into a compact plant with rapid terminal flowering. Plant Biotechnol. J. https://doi.org/10.1111/pbi.13021 (2018).

127. Brooks, C., Nekrasov, V., Lippman, Z. B. \& Van Eck, J. Efficient gene editing in tomato in the first generation using the clustered regularly interspaced short palindromic repeats/CRISPR-associated9 system. Plant Physiol. 166 1292-1297 (2014).

128. Langner, T., Kamoun, S. \& Belhaj, K. CRISPR crops: plant genome editing toward disease resistance. Annu. Rev. Phytopathol. 56, 479-512 (2018).

129. Arora, L. \& Narula, A. Gene editing and crop improvement using CRISPR-Cas9 system. Front. Plant Sci. 8, 1932 (2017)

130. Borrelli, V. M. G., Brambilla, V., Rogowsky, P., Marocco, A. \& Lanubile, A. The enhancement of plant disease resistance using CRISPR/Cas9 technology. Front. Plant Sci. 9, 1245 (2018).

131. R, C. \& HS, A. Resistance-gene-mediated defense responses against biotic stresses in the crop model plant tomato. J. Plant Pathol. Microbiol. 08, 404 (2017).

132. Zeilmaker, T. et al. Downy mildew resistant 6 and DMR6-like oxygenaSE 1 are partially redundant but distinct suppressors of immunity in Arabidopsis. Plant J. 81, 210-222 (2015)

133. Yu, W., Zhao, R., Sheng, J. \& Shen, L. SIERF2 is associated with methyl jasmonate-mediated defense response against Botrytis cinerea in tomato fruit. J. Agric. Food Chem. 66, 9923-9932 (2018).

134. Gimenez-Ibanez, S. et al. JAZ2 controls stomata dynamics during bacterial invasion. New Phytol. 213, 1378-1392 (2017).

135. Darwin, C. (ed.). On the Origin of Species 6th edn (Cambridge University Press, Cambridge, 2009).

136. Mushtaq, M. et al. CRISPR/Cas approach: a new way of looking at plant-abiotic interactions. J. Plant Physiol. 224-225, 156-162 (2018).

137. Haque, E. et al. Application of CRISPR/Cas9 genome editing technology for the improvement of crops cultivated in tropical climates: recent progress, prospects, and challenges. Front. Plant Sci. 9, 617 (2018).

138. Satpute, M. R. \& Jagdale, S. M. Color, size, volume, shape and texture feature extraction techniques for fruits: a review. Int. Res. J. Engin. Technol. 4, 703-708 (2016).

139. Li, H. et al. Tomato transcription factor SIWUS plays an important role in tomato flower and locule development. Front. Plant Sci. 8, 457 (2017).

140. Ma, X. et al. Different roles for RNA silencing and RNA processing components in virus recovery and virus-induced gene silencing in plants. J. Exp. Bot 66, 919-932 (2015). 
141. Oltman, A. E., Jervis, S. M. \& Drake, M. A. Consumer attitudes and preferences for fresh market tomatoes. J. Food Sci. 79, S2091-S2097 (2014).

142. Lin, T. et al. Genomic analyses provide insights into the history of tomato breeding. Nat. Genet. 46, 1220-1226 (2014).

143. Ballester, A. R. et al. Biochemical and molecular analysis of pink tomatoes: deregulated expression of the gene encoding transcription factor SIMYB12 leads to pink tomato fruit color. Plant Physiol. 152, 71-84 (2009).

144. Lang, Z. et al. Critical roles of DNA demethylation in the activation of ripening-induced genes and inhibition of ripening-repressed genes in tomato fruit. Proc. Natl. Acad. Sci. USA 114, E4511-E4519 (2017).

145. Ito, Y., Nishizawa-Yokoi, A., Endo, M., Mikami, M. \& Toki, S. CRISPR/Cas9mediated mutagenesis of the RIN locus that regulates tomato fruit ripening Biochem. Bioph. Res. Commun. 467, 76-82 (2015).

146. Kris-Etherton, P. M. et al. Bioactive compounds in foods: their role in the prevention of cardiovascular disease and cancer. Am. J. Med. 113, 71-88 (2002).

147. Meng, X. et al. Physiological changes in fruit ripening caused by overexpression of tomato SIAN2, an R2R3-MYB factor. Plant Physiol. Biochem. 89, 24-30 (2015)

148. Zeder, M. A. The domestication of animals. J. Anthropol. Res. 68, 161-190 (2012).

149. Zeder, M. A. in Documenting Domestication: New Genetic And Archaeological Paradigms 1st edn (eds Zeder, M. A., Bradley, D. G., Emshwiller, E. \& B. D. Smith) Ch. 13 (University of California Press, Berkeley, 2006).

150. Zsögön, A., Cermak, T., Voytas, D. \& Peres, L. E. P. Genome editing as a tool to achieve the crop ideotype and de novo domestication of wild relatives: case study in tomato. Plant Sci. 256, 120-130 (2017).

151. Soyk, S. et al. Bypassing negative epistasis on yield in tomato imposed by a domestication gene. Cell 169, 1142-1155 (2017).

152. Ledford, H. Fixing the tomato: CRISPR edits correct plant-breeding snafu. Nature 545, 394-395 (2017)

153. Zhou, J., Wang, G. \& Liu, Z. Efficient genome editing of wild strawberry genes, vector development and validation. Plant Biotechnol. J. 16, 1868-1877 (2018).

154. Kaur, N. et al. CRISPR/Cas9-mediated efficient editing in phytoene desaturase (PDS) demonstrates precise manipulation in banana CV. Rasthali genome. Funct. Integr. Genom. 18, 89-99 (2018).

155. Nakajima, I. et al. CRISPR/Cas9-mediated targeted mutagenesis in grape. PLos ONE 12, e177966 (2017).

156. Nishitani, C. et al. Efficient genome editing in apple using a CRISPR/ Cas9 system. Sci. Rep. 6, 31481 (2016).
157. Tian, S. et al. Efficient CRISPR/Cas9-based gene knockout in watermelon. Plant Cell Rep. 36, 399-406 (2017).

158. Wang, Z. et al. Optimized paired-sgRNA/Cas9 cloning and expression cassette triggers high-efficiency multiplex genome editing in kiwifruit. Plant Biotechnol. J. 16, 1424-1433 (2018).

159. Tang, T. et al. Development and validation of an effective CRISPR/Cas9 vector for efficiently isolating positive transformants and transgene-free mutants in a wide range of plant species. Front. Plant Sci. 9, 1533 (2018).

160. Xing, S. et al. CRISPR/Cas9-introduced single and multiple mutagenesis in strawberry. J. Genet Genom. 45, 685-687 (2018).

161. Roque, E. et al. Functional specialization of duplicated AP3-like genes in Medicago truncatula. Plant J. 73, 663-675 (2013).

162. Martín-Pizarro, C., Triviño, J. C. \& Posé, D. Functional analysis of the TM6 MADS-box gene in the octoploid strawberry by CRISPR/Cas9-directed mutagenesis. J. Exp. Bot. 70, 885-895 (2019).

163. Feng, J. et al. Reporter gene expression reveals precise auxin synthesis sites during fruit and root development in wild strawberry. J. Exp. Bot. 70, 563-574 (2019).

164. Sattar, M. N. et al. CRISPR/Cas9: a practical approach in date palm genome editing. Front Plant Sci. 8, 1469 (2017)

165. Zhang, $Y$. et al. Efficient and transgene-free genome editing in wheat through transient expression of CRISPR/Cas9 DNA or RNA. Nat. Commun. $\mathbf{7}$ 12617 (2016).

166. Woo, J. W. et al. DNA-free genome editing in plants with preassembled CRISPR-Cas9 ribonucleoproteins. Nat. Biotechnol. 33, 1162-1164 (2015).

167. Liang, Z. et al. Efficient DNA-free genome editing of bread wheat using CRISPR/Cas9 ribonucleoprotein complexes. Nat. Commun. 8, 14261 (2017).

168. Andersson, M. et al. Genome editing in potato via CRISPR-Cas9 ribonucleoprotein delivery. Physiol. Plant 164, 378-384 (2018).

169. Nagamangala Kanchiswamy, C., Sargent, D. J., Velasco, R., Maffei, M. E. \& Malnoy, M. Looking forward to genetically edited fruit crops. Trends Biotechnol. 33, 62-64 (2015).

170. Waltz, E. Gene-edited CRISPR mushroom escapes US regulation. Nature $\mathbf{5 3 2}$ 293 (2016).

171. Scheben, A. \& Edwards, D. Genome editors take on crops. Science 355 1122-1123 (2017).

172. Yamamoto, T. et al. Application and development of genome editing technologies to the Solanaceae plants. Plant Physiol. Biochem. 131, 37-46 (2018).

173. Callaway, E. CRISPR plants now subject to tough GM laws in European Union. Nature 560, 16 (2018). 